\title{
A New Tri-Fab Bispecific Antibody for Pretargeting Trop-2-Expressing Epithelial Cancers
}

\author{
Robert M. Sharkey ${ }^{1}$, Catharina M. van Rij ${ }^{2}$, Habibe Karacay ${ }^{1}$, Edmund A. Rossi ${ }^{3}$, Cathelijne Frielink ${ }^{2}$, Celeste Regino ${ }^{4}$, \\ Thomas M. Cardillo ${ }^{4}$, William J. McBride ${ }^{4}$, Chien-Hsing Chang ${ }^{4}$, Otto C. Boerman ${ }^{2}$, and David M. Goldenberg ${ }^{1}$ \\ ${ }^{1}$ Garden State Cancer Center, Center for Molecular Medicine and Immunology, Morris Plains, New Jersey; ${ }^{2}$ Department of Nuclear \\ Medicine, Radboud University Nijmegen Medical Centre, Nijmegen, The Netherlands; ${ }^{3}$ IBC Pharmaceuticals, Inc., Morris Plains, \\ New Jersey; and ${ }^{4}$ Immunomedics, Inc., Morris Plains, New Jersey
}

RS7 is an internalizing anti-Trop-2 pancarcinoma antibody capable of targeting most epithelial cancers. Because pretargeting strategies could improve the tumor localization of radionuclides, a new anti-Trop- $\times$ antihapten bispecific antibody for pretargeting, based on humanized RS7, was prepared and evaluated with a radiolabeled hapten-peptide in vitro and in vivo to determine whether its internalization properties would interfere with pretargeting. Methods: The anti-Trop- $\times$ antihapten bispecific antibody, TF12, was prepared using the modular dock-and-lock method. TF12 and humanized RS7 binding was assessed by cell binding assays and fluorescence-activated cell sorting analysis in a variety of human carcinoma cell lines. The internalization of TF12 was evaluated in vitro using a fluorescent TF12 conjugate or hapten-peptide and ${ }^{111}$ In-labeled TF12 and RS7. The biodistribution of TF12 and its use as a pretargeting agent with an ${ }^{111} \mathrm{In}$-labeled hapten-peptide were assessed in several human epithelial cancer xenografts. Dose optimization was examined in 2 tumor models. Results: TF12 internalizes, but a substantial fraction remained accessible on the tumor surface. Fluorescence-activated cell sorting analysis showed only a minor change in fluorescent signal when the tumor was probed with a fluorescent hapten-peptide over $4 \mathrm{~h}$, and microscopy showed substantial membrane staining when reassessed at $24 \mathrm{~h}$ after TF12 exposure. Only $40.1 \%$ of ${ }^{111}$ In-TF12 was internalized after $24 \mathrm{~h}$. In vivo, excellent tumor localization of the ${ }^{111}$ In-labeled peptide was observed in several tumor models. Conclusion: TF12 was retained sufficiently on the cell surface in several epithelial cancers, thereby making it suitable for pretargeted imaging and therapy of various Trop-2-expressing carcinomas.

Key Words: bispecific antibody; epithelial cancers; epithelial glycoprotein-1 (EGP-1); pretargeting; radioimmunodetection; Trop-2

J Nucl Med 2012; 53:1625-1632

DOI: 10.2967/jnumed.112.104364

\footnotetext{
Received Feb. 13, 2012; revision accepted May 11, 2012.

For correspondence or reprints contact: Robert M. Sharkey, Garden State Cancer Center at the Center for Molecular Medicine and Immunology, 300

The American Rd., Morris Plains, NJ 07950.

E-mail: rmsharkey@gscancer.org

Published online Sep. 5, 2012.

COPYRIGHT (C 2012 by the Society of Nuclear Medicine and Molecular Imaging, Inc.
}

A lthough radioimmunotherapy is an effective therapeutic option for follicular non-Hodgkin lymphoma (1-3), solid tumors pose challenges, and efforts continue in the search for new targets and procedures to improve imaging and therapeutic prospects (3). In this search, Trop-2 (also known as epithelial glycoprotein-1, gastric antigen 7331, and tumor-associated calcium signal transducer 2) is of interest (4). Trop-2 is a $47.8-\mathrm{kD}$ integral membrane glycoprotein that is highly expressed on most epithelial cancers originating from the breast, prostate, lungs, ovaries, gastrointestinal tract, pancreas, and skin and exhibits selective expression in some normal tissues $(5,6)$. Trop-2 expression is associated with poor prognosis, tumor aggressiveness, and metastasis (4). RS7 is an anti-Trop-2 monoclonal $\mathrm{IgG}$ antibody that targets epithelial cancers, including those of the lung, breast, colon, pancreas, and prostate (6-13). Previous studies showed that RS7 is internalized rapidly after binding Trop- 2 on the target cell surface, with an estimate of $50 \%$ internalized within $70 \mathrm{~min}$ in a breast cancer cell line, whereas even faster internalization rates were determined in a lung cancer cell line $(6,8)$. In vitro and in vivo studies showing that radiometal-labeled RS7 (e.g., ${ }^{111}$ In or ${ }^{90} \mathrm{Y}$ ) is retained at a much higher level than radioiodinated RS7 also are consistent with RS7's internalization $(10,11)$. More recently, this property led to an evaluation of RS7 as a targeting agent for drugs $(14,15)$. However, unconjugated humanized RS7 (hRS7) showed potent antibody-dependent cell-mediated cytotoxicity activity in gynecologic cancers, suggesting that some portion of hRS7 is retained on the surface to allow immune effector cell interaction.

Numerous studies have shown that various pretargeting strategies are superior to directly radiolabeled antibodies in generating high tumor-to-nontumor ratios quickly, with tumor uptake frequently rivaling that of a directly radiolabeled $\mathrm{F}\left(\mathrm{ab}^{\prime}\right)_{2}$ or even an $\operatorname{IgG}(16)$. With its pancarcinomatargeting capability, the anti-Trop- 2 antibody RS7 is an interesting antibody for pretargeting, but with previous reports indicating that $\mathrm{RS} 7$ is internalized $(6,8)$, its utility in pretargeting - for which the primary targeting agent must remain accessible for some time to allow binding of the 
secondary compound-was in question. Nevertheless, with the ease of making bispecific antibodies (bsmAbs) with the dock-and-lock technology (DNL) (17), an RS7-based antiTrop- $2 \times$ antihapten Tri-Fab bsmAb, designated TF12, was prepared (Fig. 1). In this report, the potential for using TF12 in a pretargeting setting with a radiolabeled hapten-peptide was evaluated in vitro and in vivo in various epithelial cancer cell lines. The results show that TF12 provides excellent localization of the radiolabeled hapten-peptide in vivo, illustrating that the internalization properties of this bsmAb-target combination do not impede its development for pretargeting applications.

\section{MATERIALS AND METHODS}

\section{Cell Lines}

MDA-MB-468 (breast cancer), SK-OV-3 (ovarian cancer), PC3 (prostate cancer), Calu-3 (lung cancer), HT29 (colon cancer), LS174T (colon cancer), Capan-1 (pancreatic cancer), and Raji (Burkitt lymphoma) were purchased from American Type Culture Collection.

\section{Antibodies and Other Agents}

The TF12 Tri-Fab bsmAb (molecular weight, $157 \mathrm{kDa}$ ) was prepared by the DNL procedure (17), using a humanized version of the murine RS7 as the partner with the humanized anti-HSG (histamine-succinyl-glycine) hapten antibody, 679 (Fig. 1) (18). Purified TF12, hRS7, and other antibodies that were used as controls, including veltuzumab (humanized anti-CD20 IgG (19)) and TF8 (humanized anti-CD22 [based on epratuzumab (20)] $\times$ anti-

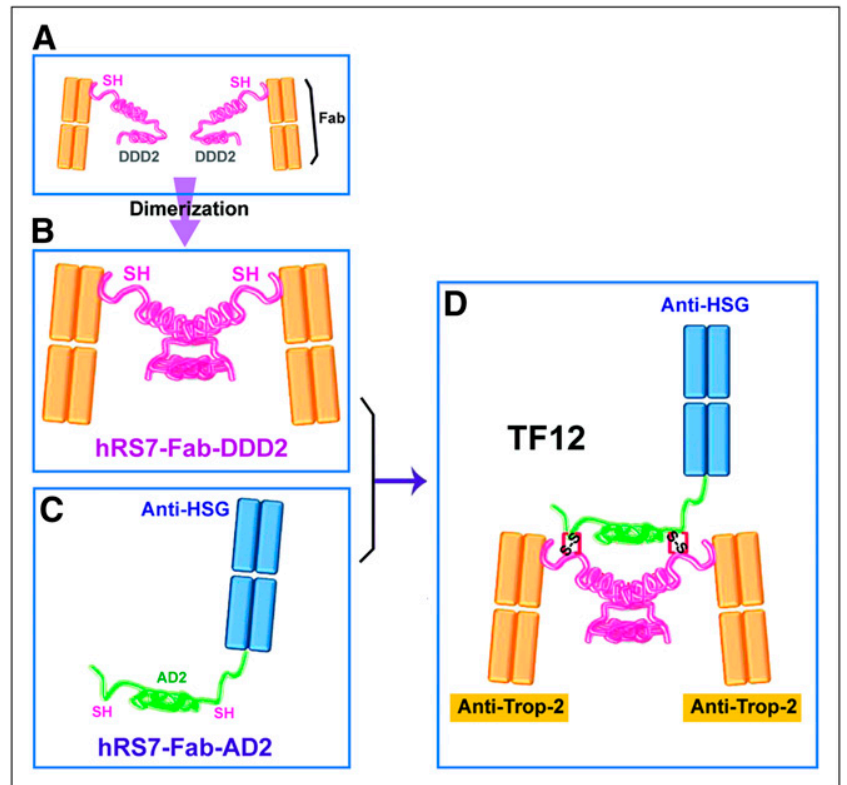

FIGURE 1. Schematic representation of TF12 DNL construct. (A) hRS7-Fab-DDD2 (DDD is dimerization and docking domain) module forms homodimers (B). h679 anti-HSG Fab is fused with AD2 sequence ( $A D$ is anchoring domain) (C). When these 2 proteins are brought together, AD2 portion docks to docking domain of DDD2 module, forming tri-Fab anti Trop- $2 \times$ anti HSG bsmAb TF12 (D). Disulfide bridges form between strategically placed cysteines to link 2 modules covalently.
HSG DNL Tri-Fab bsmAb construct), were all provided by Immunomedics, Inc., or IBC Pharmaceuticals, Inc.

Unconjugated AffiniPure goat-antihuman (GAH) IgG (heavyand light-chain-specific) and fluorescein isothiocyanate-conjugated AffiniPure GAH IgG (Fc-specific; fluorescein isothiocyanate-GAH $\mathrm{IgG}$ ) were purchased from Jackson ImmunoResearch. GAH IgG, TF12, hRS7, and veltuzumab were conjugated with AlexaFluor 488 (Invitrogen) according to the manufacturer's instructions. The AlexaFluor conjugates were designated AF-[antibody name].

IMP288, the di-HSG hapten-peptide (DOTA-D-Tyr-D-Lys(HSG)D-Glu-D-Lys(HSG)-NH2; molecular weight, 1,453 Da), was prepared and radiolabeled with ${ }^{111}$ In as described previously (21). Radioiodinated TF12 and hRS7 were made by the IODO-GEN method (22). ${ }^{125} \mathrm{I}$ and ${ }^{111}$ In were provided by either Perkin Elmer or Covidien. Instant thin-layer chromatography showed less than $5 \%$ unbound radionuclide. The immunoreactivity of radiolabeled TF12 or hRS7 was determined by Lindmo assays (23) or by sizeexclusion high-performance liquid chromatography using an excess of an anti-hRS7 idiotype antibody, as described previously (18).

A fluorescent-conjugated peptide, designated RDC017, was used to assess the accessibility of the bsmAb bound to the surface of several cell lines. RDC017 was prepared from a thiol-containing derivative of the IMP288 (supplemental data; available online only at http://jnm.snmjournals.org).

\section{Fluorescence-Activated Cell Sorting (FACS) Analysis}

FACS analysis was performed to determine hRS7 and TF12 binding to Trop-2-expressing tumor cell lines. Cell suspensions were incubated with $5 \mu \mathrm{g}$ of RS7 and TF12 AF conjugates per milliliter for $30 \mathrm{~min}$ at room temperature and were analyzed by FACS (FACSCalibur; Becton Dickinson) after being washed and fixed with $10 \%$ formalin. For each cell line, AF-veltuzumab was used as a negative control with the same procedures.

\section{Internalization Assays}

MDA-MB-468 and PC3 were incubated with TF12 $(5 \mu \mathrm{g} / \mathrm{mL})$ for $1 \mathrm{~h}$ at $4^{\circ} \mathrm{C}$. After being washed to remove the unbound antibody, fresh medium was added and incubation was continued at $37^{\circ} \mathrm{C}$. At 1,2 , and $4 \mathrm{~h}$, the presence of surface-bound TF12 was then probed using a molar excess of RDC017 for 30 min at $4{ }^{\circ} \mathrm{C}$ before being washed and then fixed with $10 \%$ buffered formalin. As an internalizing control, Raji cells were incubated with the TriFab bsmAb, TF8 (anti-CD22 $\times$ anti-HSG), using the same procedure and probing as was performed with the RDC017 peptide. Nonspecific binding was examined by incubating samples only with the fluorescent agent.

In a second approach, internalization assays using ${ }^{111}$ In-radiolabeled RS7 and TF12 with PC3 cells in culture were performed as described previously $(24,25)$. Briefly, cells were grown to confluence in 6-well plates and incubated in triplicate with either $6.15 \mathrm{ng}$ of ${ }^{111} \mathrm{In}$-radiolabeled hRS7, $4.02 \mathrm{ng}$ of ${ }^{111} \mathrm{In}-\mathrm{TF} 12$, or a combination of $4.02 \mathrm{ng}$ of ${ }^{111} \mathrm{In}$-TF12 and $1.74 \mathrm{ng}$ of unlabeled IMP288 in $2 \mathrm{~mL}$ of medium plus $0.5 \%$ bovine serum albumin (all wells received $1,200 \mathrm{~Bq}$ in $100 \mu \mathrm{L}$ of phosphate-buffered saline [PBS]). As a control, separate wells were incubated in triplicate in the presence of $2 \mu \mathrm{g}$ of unlabeled antibody before the radiolabeled product was added. After 1, 2, 4, 9, and $24 \mathrm{~h}$ of incubation at $37^{\circ} \mathrm{C}$, the cells were washed twice with cold PBS. To remove the membrane-associated fraction, the cells were incubated with an acid buffer $(0.1 \mathrm{M}$ acetic acid, $154 \mathrm{nM} \mathrm{NaCl}, \mathrm{pH} 2.6)$ for $10 \mathrm{~min}$. Then, after the cells were washed twice with PBS, they were removed 
from the plate with a cotton swab. The percentage of radioactivity in the 2 fractions, the membrane-associated and internalized fractions, with and without the addition of excess unlabeled antibody, was determined in triplicate.

\section{Microscopic Evaluation of Internalization}

An assessment of internalization of TF12 by fluorescent microscopy was performed in MDA-MB-468 cells following the procedure in the study by Pirker et al. (26). Briefly, $5 \times 10^{5} \mathrm{MDA}-$ MB-468 cells were incubated with $5 \mu \mathrm{g}$ of TF12 or control TF8 anti-CD22 per milliliter for $1 \mathrm{~h}$ at $4^{\circ} \mathrm{C}$. CD22-expressing Raji human Burkitt lymphoma cells incubated with TF8 served as a positive control for internalization, because TF8 was based on epratuzumab, a rapidly internalizing, humanized anti-CD22 antibody (20). After cells were washed with ice-cold PBS, $0.1 \%$ bovine serum albumin, and $0.1 \% \mathrm{NaN}_{3}$ to remove excess unbound $\mathrm{bsmAb}$, the cells were resuspended in fresh medium. A sample was collected immediately after washing, with the remaining cells allowed to incubate at $37^{\circ} \mathrm{C}$ for 1 or $24 \mathrm{~h}$. At the prescribed times, the cells were washed with cold medium and then probed using either the fluorescent di-HSG-peptide RDC 017 (3.2 nmol/mL) or the AF-conjugated AffiniPure GAH IgG (AF-GAH IgG). The set of cells probed with AF-GAH IgG was first fixed with $4 \%$ formalin in PBS and then permeabilized with $0.1 \%$ Triton-X-100 (5 min, room temperature) before the AF-GAH IgG was added. This procedure allowed the secondary agent to localize both surface and internalized TF12. Both the RDC017 and the AF-GAH IgG were incubated for $30 \mathrm{~min}$ at $4^{\circ} \mathrm{C}$ before the cells underwent a final wash in PBS, followed by a brief fixation in PBS-formalin. A sample was then placed on a slide for microscopic examination.

\section{Biodistribution Studies}

Studies were performed at either the Radboud University Nijmegen Medical Centre (The Netherlands) or the Center for Molecular Medicine and Immunology (United States) after these locations had received approval from their respective animal welfare committees. Mice were obtained from either Taconic or Janvier and were acclimated to laboratory conditions for at least 1 wk before tumors were implanted. The mice were housed under nonsterile standard conditions in cages with free access to food and water.

A typical study involved nude mice that were inoculated subcutaneously with $1 \times 10^{6}$ to $1 \times 10^{7}$ of MDA-MB-468, SKOV-3, PC3, or Capan-1 cells for the assessment of targeting with radiolabeled TF12 or hRS7 IgG or pretargeting with TF12. Pretargeting was also assessed using TF8 anti-CD22 bsmAb with subcutaneously grown Raji human Burkitt lymphoma cells. Once tumors were palpable, the studies were initiated (tumors generally averaged $0.1-0.3 \mathrm{~g}$ at the time of necropsy). The specific details for each study are given in the "Results" section. Animals were anesthetized and bled intracardially before being euthanized by $\mathrm{CO}_{2} / \mathrm{O}_{2}$ asphyxiation at various time points. After necropsy, tumors and organs of interest were excised, weighed, and counted in a scintillation counter. The percentage injected dose per gram $(\% \mathrm{ID} / \mathrm{g})$ was calculated on the basis of a standard of the injected product counted with the tissues.

\section{RESULTS}

\section{In Vitro Characterization of TF12 Binding}

The molecular characterization and binding properties of TF12 are given in Supplemental Figure 1. Size-exclusion high-performance liquid chromatography and sodium dodecyl sulfate polyacrylamide gel electrophoresis confirmed purity, and the binding properties of TF12 (using Biacore) to rat anti-RS7 idiotype antibody as a surrogate for Trop-2 and the hapten HSG were confirmed. The binding analysis of TF12 to MDA-MB-231 and SK-OV-3 breast and ovarian carcinoma cell lines showed a similar affinity. By flow cytometry, TF12 and hRS7 bound in a similar manner to 4 cell lines of different origin shown in Table 1. Trop- 2 expression in 2 other cell lines using hRS7 also is shown.

\section{In Vitro Assessment of Internalization}

The internalization rate of murine RS7 in Calu-3 or MBA-MD-468 previously indicated $50 \%$ of the bound antibody was internalized in approximately $1 \mathrm{~h}$ or less $(6,8)$. MDA-MB-468 cells that were preincubated with TF12 at $4^{\circ} \mathrm{C}$ before being washed and incubated at $37^{\circ} \mathrm{C}$ showed continued evidence of binding the fluorescent hapten-peptide RDC017 over $4 \mathrm{~h}$ (Fig. 2). This duration was in sharp contrast to Raji lymphoma cells incubated with TF8, an anti-CD22 bsmAb. Even after just $1 \mathrm{~h}$, the RDC017 no longer bound to the cells, suggesting TF8 had internalized completely. It is important to emphasize that this assay does not account for bsmAbs that might otherwise be released from the cells into the medium rather than being internalized. However, given the reported rapid internalization of the epratuzumab anti-CD22 antibody $(19,27,28)$, it is reasonable to assume that the inability of the peptide to bind to the Raji cells preincubated with TF8 was due to its internalization and not its release into the medium. By microscopy, MBA-MD-468 cells preincubated with TF12 and probed with RDC017 showed intense membrane localization through $24 \mathrm{~h}$, indicating that TF12 was still present on the surface (Fig. 3). However, when the cells were probed with a fluorescent-conjugated antihuman $\operatorname{IgG}$ after being fixed and permeabilized to allow the fluorescent conjugate to penetrate inside the cells, staining was seen within the cells (Figs. 3D and 3E), indicating that some portion had

TABLE 1

FACS Analysis of Trop-2 Expression in Several Epithelial Cancer Cell Lines

\begin{tabular}{lrc}
\hline \multicolumn{1}{c}{ Cell line } & hRS7 & TF12 \\
\hline MDA-468, breast & 824 & 865 \\
Capan-1, pancreatic & 66 & 71 \\
HT29, colonic & 98 & 109 \\
SK-OV-3, ovarian & 283 & 273 \\
PC3, prostate & 169 & ND \\
Calu-3, lung & 144 & ND
\end{tabular}

Trop-2 expression is given as normalized mean fluorescence intensity, subtracting corresponding negative-control MRI from TF12 or hRS7 binding.

$\mathrm{ND}=$ not determined. 


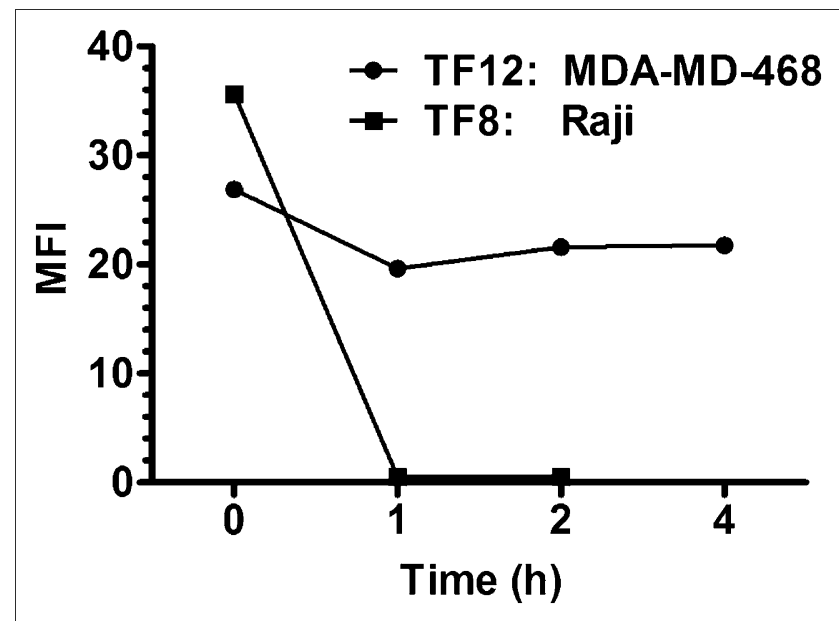

FIGURE 2. Analysis of internalization of TF12 anti-Trop-2 or TF8 anti-CD22 bsmAb in MDA-468 and Raji cells, respectively. Cells were probed with fluorescent hapten-peptide RDC017 after incubation with each bsmAb. Fluorescence, as determined by FACScan, is expressed as mean fluorescent intensity, normalizing data by subtracting mean fluorescent intensity for RDC 017 alone, which was minimal. MFI = mean fluorescent intensity.

internalized. Microscopic examination of Raji cells incubated with TF8 and probed with RDC017 immediately after the $4^{\circ} \mathrm{C}$ incubation showed a patchy distribution that became more diffuse and faint (nearly absent) when probed after a 1-h incubation at $37^{\circ} \mathrm{C}$, again suggesting that most of the anti-CD22 bsmAb had internalized (not shown).

To provide a more quantitative estimate of how much TF12 is internalized, TF12 and hRS7 were radiolabeled with ${ }^{111} \mathrm{In}$, and the fractional amount internalized over $24 \mathrm{~h}$ was evaluated in the PC3 prostate cell line. One hour after cells were incubated with the antibodies, approximately $14 \%$ of the antibody had internalized (Table 2). Over the next $8 \mathrm{~h}$, the percentage internalized increased nearly 2-fold, to $25.3 \% \pm 0.18 \%$ for the hRS7 $\mathrm{IgG}$ and to $28.3 \% \pm 2.54 \%$ for TF12. By $24 \mathrm{~h}, 40.1 \% \pm 4.99 \%$ of the TF12 bsmAb had internalized, whereas about one third
TABLE 2

Internalization of ${ }^{111}$ In-Labeled RS7, TF12, and TF12 Plus IMP288 in PC3 Cells in Culture

\begin{tabular}{cccc}
\hline & \multicolumn{3}{c}{ Percentage internalized antibody } \\
\cline { 2 - 4 } Time (h) & RS7 & TF12 & TF12 plus IMP288 \\
\hline 1 & $14.9 \pm 0.97$ & $13.6 \pm 3.09$ & $22.2 \pm 9.79$ \\
2 & $16.6 \pm 1.02$ & $16.3 \pm 1.13$ & $22.2 \pm 2.49$ \\
4 & $18.6 \pm 0.58$ & $22.8 \pm 1.47$ & $23.2 \pm 0.46$ \\
9 & $25.3 \pm 0.18$ & $28.3 \pm 2.54$ & $30.2 \pm 2.34$ \\
24 & $32.0 \pm 2.09$ & $40.1 \pm 4.99$ & $38.4 \pm 4.68$
\end{tabular}

Internalized antibody is expressed as percentage (internalized $\div$ [internalized + membrane-bound] $\times[100]$ ). In presence of excess unlabeled antibody, percentage internalized antibody at $24 \mathrm{~h}$ ranged from $2.6 \%$ to $4.1 \%$. Data are mean \pm SD.

of the hRS7 IgG was inside the cell. Thus, in this cell line, approximately $60 \%$ of the bsmAb is retained on the surface of the cells. When divalent hapten-peptide IMP288 was coadministered with radiolabeled TF12, uptake over the first $2 \mathrm{~h}$ was higher than with the bsmAb alone. These data suggest that cross-linked TF12 initiates an earlier spurt of internalization that seems to plateau, achieving the same maximum uptake as TF12 alone (not cross-linked by the hapten-peptide). As expected, preincubating cells with unlabeled antibody blocked binding of the radiolabeled antibody to Trop-2, illustrating that internalization of the radiolabeled antibody was related to its binding to Trop- 2 .

\section{Biodistribution Studies with Antibody Alone}

A biodistribution study performed in animals bearing the Capan-1 human pancreatic xenograft showed that radioiodinated TF12 cleared rapidly from the blood, with $0.15 \pm$ $0.03 \% \mathrm{ID} / \mathrm{g}$ in the blood at $24 \mathrm{~h}$ (Fig. 4), which is in agreement with the rapid clearance found with other tri-Fab bsmAbs (17). Tumor uptake peaked at $2 \mathrm{~h}$ after injection $(5.6 \pm 0.63 \% \mathrm{ID} / \mathrm{g}$ ), falling to $1.43 \pm 0.09 \% \mathrm{ID} / \mathrm{g}$ at $24 \mathrm{~h}$. Similar levels of ${ }^{125} \mathrm{I}-\mathrm{TF} 12$ were found in MBA-MD-468

FIGURE 3. Visualization of TF12 internalization. MDA-MB-468 cells were incubated for 1 $\mathrm{h}$ at $4^{\circ} \mathrm{C}$ with TF12 before being replaced with fresh medium and incubation temperature was raised to $37^{\circ} \mathrm{C}$. (A-C) Cells were then probed with fluorescent RDC017 immediately after $1 \mathrm{~h}$ of incubation at $4^{\circ} \mathrm{C}(\mathrm{A})$, after $1 \mathrm{~h}$ at $37^{\circ} \mathrm{C}(\mathrm{B})$ or after $24 \mathrm{~h}$ at $37^{\circ} \mathrm{C}(\mathrm{C})$. D and $\mathrm{E}$ represent specimens of same MDA-MB-468 cells first incubated with TF12 for $1 \mathrm{~h}$ at $4^{\circ} \mathrm{C}$, but in this case, TF12 binding was revealed using AF-GAH IgG after cells were fixed and permeabilized (binding immediately after $1 \mathrm{~h}$ of incubation at $4^{\circ} \mathrm{C}[\mathrm{D}]$ and after $1 \mathrm{~h}$ at $37^{\circ} \mathrm{C}$ $[E])$. Images were brightened and contrast was enhanced using Photoshop (Adobe).
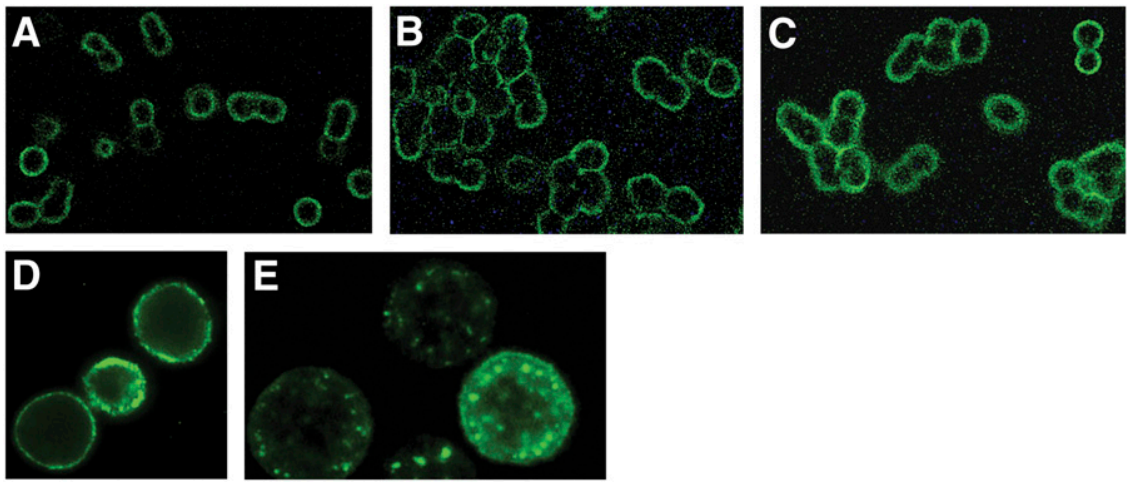


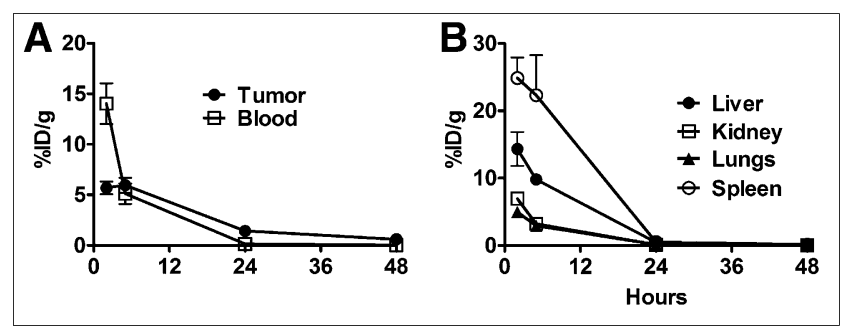

FIGURE 4. Biodistribution of ${ }^{125} \mathrm{I}-\mathrm{TF} 12$ in nude mice bearing Capan1 human pancreatic cancer xenografts. \%ID/g of tumor and blood (A) and other tissues (B).

breast and SK-OV-3 ovarian cancer cell lines at $1 \mathrm{~d}$ after injection $(0.91 \pm 0.018 \% \mathrm{ID} / \mathrm{g}$ and $1.29 \pm 0.33 \% \mathrm{ID} / \mathrm{g}$, respectively), with blood concentrations averaging $0.4 \% \mathrm{ID} / \mathrm{g}$ or less. The spleen was the only tissue that showed an unusual early uptake, averaging approximately $20 \%-25 \% \mathrm{ID} / \mathrm{g}$ at 2 and $5 \mathrm{~h}$ after injection, but like all the other normal tissues, TF12 cleared quickly, being equal to that in the liver $(\sim 0.5 \% \mathrm{ID} / \mathrm{g})$ at $24 \mathrm{~h}$.

The rapid clearance of the TF12 bsmAb and its relatively low tumor uptake were in sharp contrast to the slow blood clearance of the hRS7 IgG and its higher uptake. For example, in PC3-bearing nude mice, tumor uptake of ${ }^{125} \mathrm{I}$-hRS7 on day 3 was $7.3 \pm 2.3 \% \mathrm{ID} / \mathrm{g}$, whereas the concentration in the blood was $8.1 \pm 1.1 \% \mathrm{ID} / \mathrm{g}$. However, in PC3-bearing mice given ${ }^{111} \mathrm{In}-\mathrm{hRS} 7$, tumor uptake was $36.5 \pm 13.3 \% \mathrm{ID} / \mathrm{g}$, whereas the blood concentration was similar to radioiodinated $\mathrm{hRS} 7 \mathrm{IgG}$ on day 3 . These results are indicative of an internalizing antibody binding to a stable chelate-radiometal, where the percentage uptake accounts for the cumulative binding of hRS7, its internalization, and entrapment of the ${ }^{111} \mathrm{In}$ activity over time. However, the uptake of radioiodinated $\operatorname{IgG}$ or bsmAb is expected to reveal only the amount of antibody that remains accessible (i.e., has not been internalized and catabolized).

TABLE 3

Biodistribution of Pretargeted ${ }^{111}$ In-IMP288 in MDA-468, SK-OV-3, and PC3 at 3 Hours After Injection

\begin{tabular}{lccc}
\hline \multicolumn{1}{c}{ Organ } & MBA-MD-468 & SK-OV-3 & PC3 \\
\hline Tumor & $14.8 \pm 3.4$ & $13.6 \pm 2.6$ & $9.8 \pm 1.6$ \\
Blood & $0.02 \pm 0.00$ & $0.04 \pm 0.02$ & $0.16 \pm 0.02$ \\
Spleen & $0.17 \pm 0.03$ & $0.56 \pm 0.34$ & $0.31 \pm 0.07$ \\
Kidney & $2.45 \pm 0.55$ & $2.51 \pm 0.64$ & $1.49 \pm 0.29$ \\
Liver & $0.10 \pm 0.02$ & $0.20 \pm 0.06$ & $0.21 \pm 0.03$ \\
Tumor to blood & $1,037 \pm 303$ & $378 \pm 126$ & $62 \pm 4$ \\
Tumor weight $(g)$ & $0.06 \pm 0.02$ & $0.20 \pm 0.09$ & $0.07 \pm 0.01$ \\
\hline
\end{tabular}

Nude mice were injected intravenously with $79 \mu \mathrm{g}(0.5 \mathrm{nmol})$ of 125I-TF12 (0.37 MBq) and $0.025 \mathrm{nmol}$ of ${ }^{111} \mathrm{In}$-IMP288, with exception of PC3 studies, for which $462 \mu \mathrm{g}(\sim 3 \mathrm{nmol})$ of ${ }^{125} \mathrm{I}-\mathrm{TF} 12$ and $0.11 \mathrm{nmol}$ of ${ }^{111} \mathrm{In}$-IMP288 were injected. In all cases, ${ }^{111} \mathrm{In}-$ IMP288 was given at $16 \mathrm{~h}$ after TF12 injection. Data are expressed as $\% \mathrm{ID} / \mathrm{g}$ (mean $\pm \mathrm{SD} ; n=5$ ).

\section{Pretargeting Studies}

Despite in vitro evidence that some portion of TF12 is internalized, biodistribution studies in mice bearing different subcutaneous tumor xenografts and pretargeted the day before with TF12 showed a reasonably high $(\sim 10-15 \% \mathrm{ID} / \mathrm{g})$ uptake of the ${ }^{111}$ In-labeled peptide in the tumors (Table 3 ). Tissue uptake and blood concentrations were low, with tumor-to-blood ratios ranging from approximately 60:1 to $1,000: 1$.

Additional studies examined several doses of TF12 (40, 79 , and $158 \mu \mathrm{g}$ ) given with a fixed amount of IMP288 (0.026 nmol; TF12-to-IMP288 mol ratios, 10, 20, and 40:1) in the SK-OV-3 and MDB-MD-468 models (Fig. 5). ${ }^{111} \mathrm{In}-\mathrm{IMP} 288$ uptake in SK-OV-3 at $3 \mathrm{~h}$ increased from 5.3 $\pm 1.2 \% \mathrm{ID} / \mathrm{g}$ at the $10: 1$ ratio to as high as $21.3 \pm 6.2 \% \mathrm{ID} / \mathrm{g}$ at the 40:1 ratio. Tumor uptake for TF12 in these animals (as measured by ${ }^{125}$ I-TF12 added to TF12) was similar in the groups, ranging from 1.1 to $1.5 \% \mathrm{ID} / \mathrm{g}$. In contrast, MDAMB-468 uptake was only modestly increased from $11.6 \pm$ 1.9 to $15.2 \pm 3.3 \% \mathrm{ID} / \mathrm{g}$ over this same range of $\mathrm{TF} 12$. Interestingly, tumor uptake of the ${ }^{125} \mathrm{I}-\mathrm{TF} 12$ was nearly 2-fold higher in the MDA-MB-468 xenografts than in the SK-OV-3 tumors, yet the breast cancer xenograft did not have a higher IMP288 uptake commensurate with the higher TF12 level. By $24 \mathrm{~h}$, tumor uptake for the ${ }^{111}$ In-IMP288 plateaued in both models, even though the uptake of the ${ }^{125}$ I-TF12 decreased nearly 2 - to 4 -fold.

These targeting results are in sharp contrast to those obtained with the TF8 anti-CD22 bsmAb in BALB/c nude mice bearing CD22-expressing Ramos or Raji tumors. In this study, animals were given $0.25 \mathrm{nmol}(40 \mu \mathrm{g})$ of TF8 followed $24 \mathrm{~h}$ later with $0.025 \mathrm{nmol}$ of ${ }^{111}$ In-IMP288. Three hours later, uptake was just $0.17 \pm 0.07 \% \mathrm{ID} / \mathrm{g}$ in Raji tumors and $0.15 \pm 0.08$ in Ramos $(n=4$ in each). The low tumor uptake was predicted by the earlier in vitro studies that indicated efficient internalization within $1 \mathrm{~h}$. Thus, even though tumor-to-blood ratios were high (e.g., averaging 38:1 in Raji-bearing mice), the low tumor uptake indicated unfavorable pretargeting with this bsmAb.

\section{DISCUSSION}

Pretargeting procedures for localizing radionuclides are attractive, because they are able to localize the radiolabeled product (e.g., hapten-peptide or biotin) quickly, developing high tumor-to-non-tumor ratios rapidly, and often with a tumor uptake that can rival a directly radiolabeled IgG or $\mathrm{F}\left(\mathrm{ab}^{\prime}\right)_{2}$ fragment (16). Bispecific antibody pretargeting procedures have typically relied on the bsmAb's natural clearance from the blood and tissues, requiring a delay of several days before the hapten-peptide is given (16). It is imperative that the bsmAb remain accessible in the tumor (e.g., not internalized) in order for the radiolabeled haptenpeptide to be localized. Thus, if the pretargeting agent binds an antigen that triggers internalization, this target likely would not be suitable for pretargeting. For example, anti- 
FIGURE 5. Optimizing IMP288 uptake in SK-OV-3 human ovarian cancer and MDAMB-468 human breast cancer xenografts in nude mice. Animals ( $n=5 /$ interval) were given 40, 79, or $158 \mu \mathrm{g}$ of TF12 (containing trace ${ }^{125}$ I-TF12), and then $16 \mathrm{~h}$ later they received $0.026 \mathrm{nmol}(\sim 40 \mu \mathrm{Ci})$ of ${ }^{111} \mathrm{In}-$ IMP288. Necropsy was performed at 3 and $24 \mathrm{~h}$. Tumor and blood concentrations are shown for ${ }^{111} \mathrm{In}-\mathrm{IMP}-288$ (bars) and $125 \mathrm{I}$ TF12 (in parentheses) at $3 \mathrm{~h}(\mathrm{~A})$ and $24 \mathrm{~h}$ (B). Tumors averaged between 0.1 and $0.3 \mathrm{~g}$.

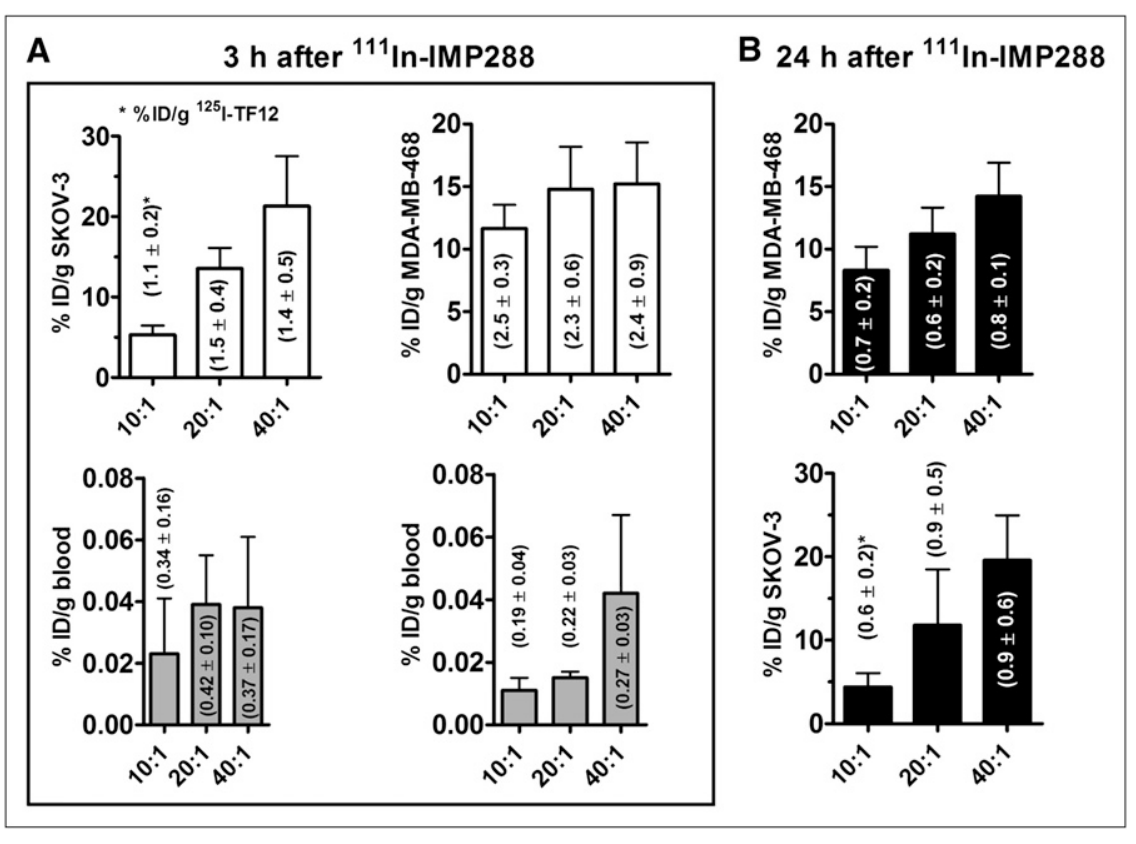

CD22 antibodies are known to internalize rapidly, making these antibodies attractive candidates for targeting drugs or toxins that exert their effects intracellularly (28-33). Pantelias et al. (34) compared the pretargeting of 3 different antigens associated with hematopoietic malignancies, anti-CD20, CD22, and HLA-DR, finding that the anti-CD22 pretargeting agent had the lowest uptake of the 3 conjugates. However, CD22 also had the lowest density of the 3 antigens in the various cell lines examined, and thus this result may have merely reflected the lower antigen density. Herein, we included an evaluation of the TF8 tri-Fab bsmAb that binds to CD22, suspecting its rapid internalization would affect its utility in pretargeting. We first noted that in vitro, the TF8 anti-CD22 bsmAb was no longer accessible within just $1 \mathrm{~h}$ of its binding, and then in vivo, no appreciable localization of the radiolabeled hapten-peptide was observed. Thus, we agree that bsmAbs to CD22 are not suitable for pretargeting, not because they are insufficiently expressed on target cells but because the bsmAbs are efficiently internalized. Instead, we previously recommended that the TF4 anti-CD20 tri-Fab bsmAb be used for pretargeting B-cell malignancies (35). In those studies, ${ }^{111}$ In-TF4 had a substantially higher uptake in tumor xenografts than radioiodinated TF4, suggesting some internalization over the 1-d pretargeting interval.

Although antibody-based therapeutics (unconjugated and conjugated) are widely studied in hematologic malignancies, it has been more challenging to find suitable targets for epithelial cancers (3). In this regard, Trop-2 represents a promising new target for cancer, being expressed on the surface of many carcinomas (4). Early in its development, the anti-Trop-2 antibody RS7 was reported to internalize, and therefore most studies have focused on developing targeting strategies that take advantage of RS7's internalization properties $(7,10,14,36)$. Shih et al. (8) examined internalization in MDA-MD-468 cells in vitro over $30 \mathrm{~min}$ and reported an extrapolated internalization rate of $0.0147 \mathrm{~min}^{-1}$, indicating that under saturating conditions, surface-bound antibody will be internalized over approximately $70 \mathrm{~min}$. Other studies in the Calu-3 human lung cancer cell line suggested an even faster rate of internalization, with most of the antibody being internalized within $45 \mathrm{~min}(6)$. This rate compares with that of the antiCD22 antibody, for which $50 \%$ of the antibody was reported to be internalized within $10 \mathrm{~min}$, with data indicating that nearly all the antibody had internalized within $2 \mathrm{~h}$ (28). In vivo studies comparing RS7 labeled with a radiometal with RS7 labeled by the more traditional noncovalent radioiodination procedures showed a substantially higher tumor retention when the antibody was labeled with a residualizing radionuclide, supporting the internalization capability of the RS7 antibody $(7,10,11)$. Thus, these data prompted interest in developing a pretargeting procedure for Trop-2 localization. However, given the presence of Trop-2 in many carcinomas, and because the modular DNL method greatly simplifies bsmAb preparation (17), TF12 was prepared for evaluation.

Our findings with the hRS7 IgG and TF12 bsmAb indicate that despite some initial internalization, sufficient residual bsmAb remains on the surface of tumor cells to capture the hapten-peptide after an approximate 1-d delay. Some of the in vitro studies were performed with the washed cells placed in fresh medium before probing with the fluorescent hapten-peptide. Thus, it is unlikely that reexpression of antigen with reengagement by the bsmAb contained in the medium explained the continued presence of TF12 on the cell surface. Because TF12 clears so quickly from the blood, it is equally unlikely that there would be considerable replenishing of the bsmAb after the internal- 
ization process had occurred. Therefore, although there have been reports that internalization is nearly complete within approximately $1 \mathrm{~h}$, our studies with TF12 and hRS7 radioconjugates found that a smaller portion of the antibody, perhaps as low as $10 \%-20 \%$, is internalized over the first hour and that internalization proceeds at a much slower rate thereafter (i.e., it is not linear). Indeed, it appears that after an initial spurt of internalization, some cell lines had only about $50 \%$ of the antibody internalized over $24 \mathrm{~h}$.

These observations are important, because antibodies are often characterized as being internalizing or noninternalizing when in fact there are varying rates among antibodies that are actively internalized and rates for those passively taken into the cell. At least in the case of TF12, the in vitro studies using the fluorescent hapten-peptide were helpful for illustrating the continued capacity to bind the haptenpeptide after several hours and even $1 \mathrm{~d}$. This type of assay should be useful for predicting which bsmAb would have suitable surface retention for in vivo targeting, but it is unlikely it will be useful for predicting the actual quality of a given pretargeting agent, because there are other parameters that affect tumor localization (37). With recent clinical studies using the TF2 anti-CEACAM5 tri-Fab bsmAb also finding that the bsmAb is cleared sufficiently within just 1 $\mathrm{d}$ to allow the efficient localization of the radiolabeled hapten-peptide $(38,39)$, we are encouraged to continue developing the TF12 bsmAb for future clinical evaluation.

\section{CONCLUSION}

TF12 is a suitable bsmAb for pretargeting different cancers that express Trop-2, primarily because after an initial spurt of internalization, sufficient bsmAb remains on the surface to allow efficient capture of the hapten-peptide. With recent preclinical studies showing hRS7 targeting of prostate cancer (13), and with the need for developing better imaging procedures for this disease, this clinical indication should be considered. However, hRS7-SN-38 drug conjugates also have been proven to be effective in several cancer cell lines, including lung, pancreatic, colorectal, and breast $(6,7,12,14,15)$. Thus, developing a highly sensitive imaging procedure for these cancers, perhaps to be used in concert with the therapeutic agent, is of interest as well.

\section{DISCLOSURE STATEMENT}

The costs of publication of this article were defrayed in part by the payment of page charges. Therefore, and solely to indicate this fact, this article is hereby marked "advertisement" in accordance with 18 USC section 1734.

\section{ACKNOWLEDGMENTS}

We thank Jayson Jebsen, Ali Mostafa, Lenka Muskova, Preeti Trisal, Anke Reuser, Gerben Franssen, Annemarie Eek, Kitty Lemmens, and Bianca Lemmers for their excellent technical assistance. This study was supported in part by NJ Cancer Commission grant 10-8-CCR-EO and the Dutch Cancer Society grant KUN-2010-4820. Edmund A. Rossi, Celeste Regino, Thomas M. Cardillo, William J. McBride, Chien-Hsing Chang, and David M. Goldenberg are employed or have financial interests in Immunomedics, Inc., or IBC Pharmaceuticals, Inc. No other potential conflict of interest relevant to this article was reported.

\section{REFERENCES}

1. Hohloch K, Delaloye AB, Windemuth-Kieselbach C, et al. Radioimmunotherapy confers long-term survival to lymphoma patients with acceptable toxicity: registry analysis by the International Radioimmunotherapy Network. J Nucl Med. 2011;52:1354-1360.

2. Illidge T, Morschhauser F. Radioimmunotherapy in follicular lymphoma. Best Pract Res Clin Haematol. 2011;24:279-293.

3. Sharkey RM, Goldenberg DM. Cancer radioimmunotherapy. Immunotherapy. 2011;3:349-370.

4. Cubas R, Li M, Chen C, Yao Q. Trop2: a possible therapeutic target for late stage epithelial carcinomas. Biochim Biophys Acta. 2009;1796:309-314.

5. Stepan LP, Trueblood ES, Hale K, Babcook J, Borges L, Sutherland CL. Expression of Trop2 cell surface glycoprotein in normal and tumor tissues: potential implications as a cancer therapeutic target. J Histochem Cytochem. 2011;59:701-710.

6. Stein R, Basu A, Chen S, Shih LB, Goldenberg DM. Specificity and properties of MAb RS7-3G11 and the antigen defined by this pancarcinoma monoclonal antibody. Int J Cancer. 1993;55:938-946.

7. Govindan SV, Stein R, Qu Z, et al. Preclinical therapy of breast cancer with a radioiodinated humanized anti-EGP-1 monoclonal antibody: advantage of a residualizing iodine radiolabel. Breast Cancer Res Treat. 2004;84:173-182.

8. Shih LB, Xuan H, Aninipot R, Stein R, Goldenberg DM. In vitro and in vivo reactivity of an internalizing antibody, RS7, with human breast cancer. Cancer Res. 1995;55:5857s-5863s

9. Stein R, Blumenthal R, Sharkey RM, Goldenberg DM. Comparative biodistribution and radioimmunotherapy of monoclonal antibody RS7 and its $\mathrm{F}(\mathrm{ab})_{2}$ in nude mice bearing human tumor xenografts. Cancer. 1994;73:816-823.

10. Stein R, Chen S, Haim S, Goldenberg DM. Advantage of yttrium-90-labeled over iodine-131-labeled monoclonal antibodies in the treatment of a human lung carcinoma xenograft. Cancer. 1997;80:2636-2641.

11. Stein R, Goldenberg DM, Thorpe SR, Mattes MJ. Advantage of a residualizing iodine radiolabel for radioimmunotherapy of xenografts of human non-small-cell carcinoma of the lung. J Nucl Med. 1997;38:391-395.

12. Stein R, Govindan SV, Chen S, et al. Successful therapy of a human lung cancer xenograft using MAb RS7 labeled with residualizing radioiodine. Crit Rev Oncol Hematol. 2001;39:173-180.

13. van Rij CM, Sharkey RM, Goldenberg DM, et al. Imaging of prostate cancer with immuno-PET and immuno-SPECT using a radiolabeled anti-EGP-1 monoclonal antibody. J Nucl Med. 2011;52:1601-1607.

14. Cardillo TM, Govindan SV, Sharkey RM, Trisal P, Goldenberg DM. Humanized anti-Trop-2 IgG-SN-38 conjugate for effective treatment of diverse epithelial cancers: preclinical studies in human cancer xenograft models and monkeys. Clin Cancer Res. 2011;17:3157-3169.

15. Sharkey RM, Karacay H, Govindan SV, Goldenberg DM. Combination radioimmunotherapy and chemoimmunotherapy involving different or the same targets improves therapy of human pancreatic carcinoma xenograft models. Mol Cancer Ther. 2011;10:1072-1081.

16. Goldenberg DM, Chatal JF, Barbet J, Boerman O, Sharkey RM. Cancer imaging and therapy with bispecific antibody pretargeting. Update Cancer Ther. 2007; 2:19-31.

17. Rossi EA, Goldenberg DM, Cardillo TM, McBride WJ, Sharkey RM, Chang CH. Stably tethered multifunctional structures of defined composition made by the dock and lock method for use in cancer targeting. Proc Natl Acad Sci USA. 2006; 103:6841-6846.

18. Sharkey RM, McBride WJ, Karacay H, et al. A universal pretargeting system for cancer detection and therapy using bispecific antibody. Cancer Res. 2003;63: $354-363$.

19. Goldenberg DM, Rossi EA, Stein R, et al. Properties and structure-function relationships of veltuzumab (hA20), a humanized anti-CD20 monoclonal antibody. Blood. 2009;113:1062-1070. 
20. Leung SO, Goldenberg DM, Dion AS, et al. Construction and characterization of a humanized, internalizing, B-cell (CD22)-specific, leukemia/lymphoma antibody, LL2. Mol Immunol. 1995;32:1413-1427.

21. Karacay H, Sharkey RM, Gold DV, et al. Pretargeted radioimmunotherapy of pancreatic cancer xenografts: TF10-90 Y-IMP-288 alone and combined with gemcitabine. J Nucl Med. 2009;50:2008-2016.

22. Fraker PJ, Speck JC Jr. Protein and cell membrane iodinations with a sparingly soluble chloroamide, 1,3,4,6-tetrachloro-3a,6a-diphrenylglycoluril. Biochem Biophys Res Commun. 1978;80:849-857.

23. Lindmo T, Boven E, Cuttitta F, Fedorko J, Bunn PA Jr. Determination of the immunoreactive fraction of radiolabeled monoclonal antibodies by linear extrapolation to binding at infinite antigen excess. J Immunol Methods. 1984;72:77-89.

24. de Jong M, Breeman WA, Bakker WH, et al. Comparison of ${ }^{111}$ In-labeled somatostatin analogues for tumor scintigraphy and radionuclide therapy. Cancer Res. 1998;58:437-441.

25. van Oosterhout YV, van den Herik-Oudijk IE, Wessels HM, de Witte T, van de Winkel JG, Preijers FW. Effect of isotype on internalization and cytotoxicity of CD19-ricin A immunotoxins. Cancer Res. 1994;54:3527-3532.

26. Pirker R, FitzGerald DJ, Hamilton TC, et al. Characterization of immunotoxins active against ovarian cancer cell lines. J Clin Invest. 1985;76:1261-1267.

27. Carnahan J, Wang P, Kendall R, et al. Epratuzumab, a humanized monoclonal antibody targeting CD22: characterization of in vitro properties. Clin Cancer Res. 2003;9:3982S-3990S.

28. Shih LB, Lu HH, Xuan H, Goldenberg DM. Internalization and intracellular processing of an anti-B-cell lymphoma monoclonal antibody, LL2. Int J Cancer. 1994;56:538-545.

29. Ghetie V, Engert A, Schnell R, Vitetta ES. The in vivo anti-tumor activity of immunotoxins containing two versus one deglycosylated ricin A chains. Cancer Lett. 1995;98:97-101.
30. Newton DL, Hansen HJ, Mikulski SM, Goldenberg DM, Rybak SM. Potent and specific antitumor effects of an anti-CD22-targeted cytotoxic ribonuclease: potential for the treatment of non-Hodgkin lymphoma. Blood. 2001;97:528-535.

31. Kreitman RJ, Pastan I. Immunotoxins in the treatment of hematologic malignancies. Curr Drug Targets. 2006;7:1301-1311.

32. Polson AG, Calemine-Fenaux J, Chan $\mathrm{P}$, et al. Antibody-drug conjugates for the treatment of non-Hodgkin's lymphoma: target and linker-drug selection. Cancer Res. 2009;69:2358-2364.

33. Sharkey RM, Govindan SV, Cardillo TM, Goldenberg DM. Epratuzumab-SN-38: a new antibody-drug conjugate for the therapy of hematological malignancies. Mol Cancer Ther. 2012;11:224-234.

34. Pantelias A, Pagel JM, Hedin N, et al. Comparative biodistributions of pretargeted radioimmunoconjugates targeting CD20, CD22, and DR molecules on human B-cell lymphomas. Blood. 2007;109:4980-4987.

35. Sharkey RM, Karacay H, Johnson CR, et al. Pretargeted versus directly targeted radioimmunotherapy combined with anti-CD20 antibody consolidation therapy of non-Hodgkin lymphoma. J Nucl Med. 2009;50:444-453.

36. Chang $\mathrm{CH}$, Gupta P, Michel R, et al. Ranpirnase (frog RNase) targeted with a humanized, internalizing, anti-Trop- 2 antibody has potent cytotoxicity against diverse epithelial cancer cells. Mol Cancer Ther. 2010;9:2276-2286.

37. van Schaijk FG, Oosterwijk E, Molkenboer-Kuenen JD, et al. Pretargeting with bispecific anti-renal cell carcinoma x anti-DTPA(In) antibody in 3 RCC models. J Nucl Med. 2005;46:495-501.

38. Sharkey RM, Rossi EA, McBride WJ, Chang CH, Goldenberg DM. Recombinant bispecific monoclonal antibodies prepared by the dock-and-lock strategy for pretargeted radioimmunotherapy. Semin Nucl Med. 2010;40:190-203.

39. Schoffelen R, Boerman O, van der Graff W, et al. Phase I clinical study of the feasibility of pretargeted radioimmunotherapy (PT-RAIT) in patients with colorectal cancer (CRC): first results [abstract]. J Nucl Med. 2011;52(suppl 1):358. 